\title{
Review of: "Quo Vadis? Carbon Peak and Emission Network for China in the Post-Pandemic Era"
}

\author{
Fang Wang ${ }^{1}$ \\ 1 Sichuan Agricultural University
}

Potential competing interests: The author(s) declared that no potential competing interests exist.

Achieving carbon peak is essential for China achieving carbon neutrality by 2060 , and is also important for addressing climate change worldwide. The author prepares a comprehensive analysis on this regard. In general, this paper is interesting and important. The authors present a comprehensive analysis on carbon peak based on the detailed datasets and considering some important recent changes such as the so-called "new normal economy" and the outbreak of COVID-19 pandemic as well as slowing rate of decreasing carbon intensity for China. Overall, this is an interesting and reasonably well-written paper. The study can be potentially important for policy implication for achieving the carbon peak for China and other emerging economies. Here are some suggestions that authors require to pay attention to. I hope these details can help authors improve the article.

1. The title "Quo vadis? Carbon peak and emission network for China in the post-pandemic era" doesn't seem to reflect the focus of the study as the emission network analysis is from the perspective of emission mitigation for achieving carbon peak. Therefore, I suggest the author to revise the title for better reflecting the focus of the study.

2. - Achieving carbon peak by 2030 would be the condition of achieving carbon natural by 2060 for China. Therefore, provide info about carbon neutrality policy in China in introduction section.

3. - There are many important studies (related to the study) that were not cited in the manuscript. More comprehensive review of the published literature is needed.

4. Rewrite your conclusion section. Clearly presents your main results and provide recommendations for further investigations in conclusion section. Also, clearly state the limitations of the study.

5. References should be revised, expanded and listed in chronological order.

6. I also recommend editing your paper according to the instructions for authors in the journal website. 